\title{
WAYFINDING, ROBOTICS AND HOSPITALS
}

\author{
by \\ Kim Kamaljeet Ghattoura \\ Bachelor of Design, Toronto Ontario, April 2014 \\ A Major Research Paper \\ Presented to Ryerson University \\ in partial fulfillment of the \\ requirements for the degree of \\ Master of Digital Media \\ In the Yeates School of Graduate Studies \\ Toronto, Ontario, Canada, 2016 \\ (C) Kim Kamaljeet Ghattoura, 2016
}




\section{Author's Declaration}

I hereby declare that I am the sole author of this MRP. This is a true copy of the MRP, including any required final revisions.

I authorize Ryerson University to lend this MRP to other institutions or individuals for the purpose of scholarly research.

I further authorize Ryerson University to reproduce this MRP by photocopying or by other means, in total or in part, at the request of other institutions or individuals for the purpose of scholarly research.

I understand that my MRP may be made electronically available to the public.

Kim Kamaljeet Ghattoura 


\title{
Abstract
}

\author{
WAYFINDING, ROBOTICS AND HOSPITALS \\ Master of Digital Media, 2016 \\ Kim Kamaljeet Ghattoura \\ Master of Digital Media, Ryerson University
}

This paper will give a better understanding on how complex healthcare facilities such as hospitals are using robotic technologies that incorporate superior Wayfinding systems to carry out tasks and obtain information which originally, were conducted by healthcare workers, nurses and or doctors. This paper will also address the ethical concerns that people have by incorporating these robotic technologies such as: third parties being able to hack into these technologies and collect confidential information from patients, healthcare workers and nurses possibly loosing their jobs to hospital robots and whether or not hospital robots are in fact, safe to be operating within a environment where peoples lives are on the line. 


\section{Acknowledgements}

This major research paper would not have been possible without the support and guidance of my supervisor Dr. Richard Lachman from Ryerson University. I would also like to thank my wonderful parents and family for their continuous support and guidance through my academic and creative career. My inspiration Daniel Libeskind and Jessica Walsh. I would also like to thank the following: Mark Saxler, Sonya Taccone, Michael Carter for their guidance and help throughout this thesis. 


\section{TABLE OF CONTENTS}

Authors Declaration - ii

Abstract - iii

Acknowledgments - iv

\section{Chapter One - Evolution of Wayfinding}

1.0 What is Wayfinding? - 2

1.1 Why is Wayfinding important? - 2

1.2 Traditional Wayfinding methods and systems -4

1.3 Wayfinding digital transitions - 8

1.4 Wayfinding digital technologies such as GPS and kiosk screens - 9

\section{Chapter Two - Robotic Technologies}

2.0 Introduction to robotics -15

2.1 Technological advancements and examples of significant robotic advancements -18

2.2 Common components of robotics - 22

2.3 Robotic technologies with Wayfinding for hospitals - 25

2.4 The technology of RFID, RFID tags and Wi-Fi - 31

Chapter Three - Ethical Concerns about Robotic Technologies in Hospitals

3.0 They are just like one of us, apart of our team - 36

3.1 Will these robots replace healthcare workers and nurses? Will these robots take the humanity out of healthcare? - 40

3.2 How easy is it to hack into these robots and obtain confidential information? - 47

3.3 Are robotic technologies like TUG and Homer safe to be operating in a environment where peoples lives are on the line? - 51

\section{Chapter Four - Future Projections, Concerns and Conclusions}

4.0 The future of robots in hospitals -55

4.1 Conclusions -58

\section{References - 61}




\section{Introduction}

Throughout history, there have been vast changes in the types of technologies that are being used and associated with Wayfinding systems such as robotics. Today, robotic technologies are widely used by companies and organizations to complete various tasks and procedures that are commonly completed by people. However, now, new and advanced hospitals are looking to incorporate innovative robotic technologies with superior Wayfinding systems to complete similar tasks that are commonly carried out by nurses and healthcare workers.

The thesis "Wayfinding, robotics and hospitals" includes four chapters. The first chapter is about the evolution of Wayfinding. This chapter will focus on the transition from traditional Wayfinding systems into new advanced Wayfinding technologies. The second chapter is about robotics. This chapter will illustrate past innovations in robotics and their connection to hospital robots today. The third chapter is about the ethical concerns about robotic technologies in hospitals. This chapter will focus on the impact robots are having on current hospital staff and if these robots are in fact, safe to be operating in hospitals. The last and final chapter is about future projections on robotics in hospitals. This chapter will showcase the types of characteristics that designers and engineers are predicting robots in hospitals to acquire in the upcoming years. 


\section{Chapter One - Evolution of Wayfinding}

\subsection{What is Wayfinding?}

Wayfinding is a term that is often interpreted in various distinct contexts because Wayfinding does in fact, serve several different spaces and structures that require unique customized systems. However, the essence of Wayfinding can be explained by the following:

The heart of a civilization throbs wherever people come together to work, play, shop, study, perform, worship, or even just interact. Crowded into bustling spaces, they share the richness and diversity of human experience as well as it challenges. In these spaces people may 'find their way' in the existential sense, but they also become overwhelmed or disoriented if they physically lose their way. Wayfinding design provides guidance and the means to help people feel at east in their surrounds (Gibson, 2009:12).

\subsection{Why is Wayfinding important?}


Various spaces are filled with a lot of information, which include signs and symbols. The job of a Wayfinding designer is to take this information and create a Wayfinding system that not only allows clear navigation, but also allows the best possible experience to an individual (Gibson, 2009). Wayfinding is implemented in our everyday lives, but we are often unaware of how important it really is until we are forced to rely on the infrastructure that has been put in place to guide us. Every structure requires a navigation system to allow visitors or residents to get from one place to another. However, in many cases it is challenging to create a unique and successful Wayfinding system that allows individuals to move smoothly from one destination to another without creating additional disturbances to other navigation systems present.

In many instances Wayfinding navigation systems are designed after structures are built. This contrasts with more modern structures, which are being built by focusing on how people will interact and navigate through the spaces (Hayzlett, 2015). Since these new moderns structures are made to reinforce better and easy navigation for the public, Wayfinding systems are now are being implemented in design plans and blueprints before structures are built.

Great Way-finding systems combine both explicit and implicit signs that allow 
for more accurate and clear communication (Gibson, 2009). As infrastructure grows and expands throughout the world, designers are now more then ever, required to create Wayfinding systems that produce accurate and immediate information to foreign visitors or everyday residents.

There are many complex spaces that are hard to navigate through such as airports, shopping malls and large educational institutions such as universities. However, "a hospital is a unique building type in terms of the high level of complexity in circulation patterns, constant use and diverse technical system. A hospital delivers healthcare services to patients who may stay in overnight accommodations or may visit briefly for specific care" (Reffat, 1). Designers and engineers are constantly trying to come up with superior Wayfinding systems that can be implemented in hospitals: a complex facility that is utilized 24 hours a day and 7 days a week.

\subsection{Traditional Wayfinding methods and systems}

Wayfinding navigation systems have been implemented in various structures and spaces for numerous years throughout history. We continuously use these navigation systems in our everyday lives to move from point $A$ to point $B$. 
However, before new digital and robotic technologies emerged, there have been many dominant, influential and traditional Wayfinding navigation systems that have been used in many structures and spaces.

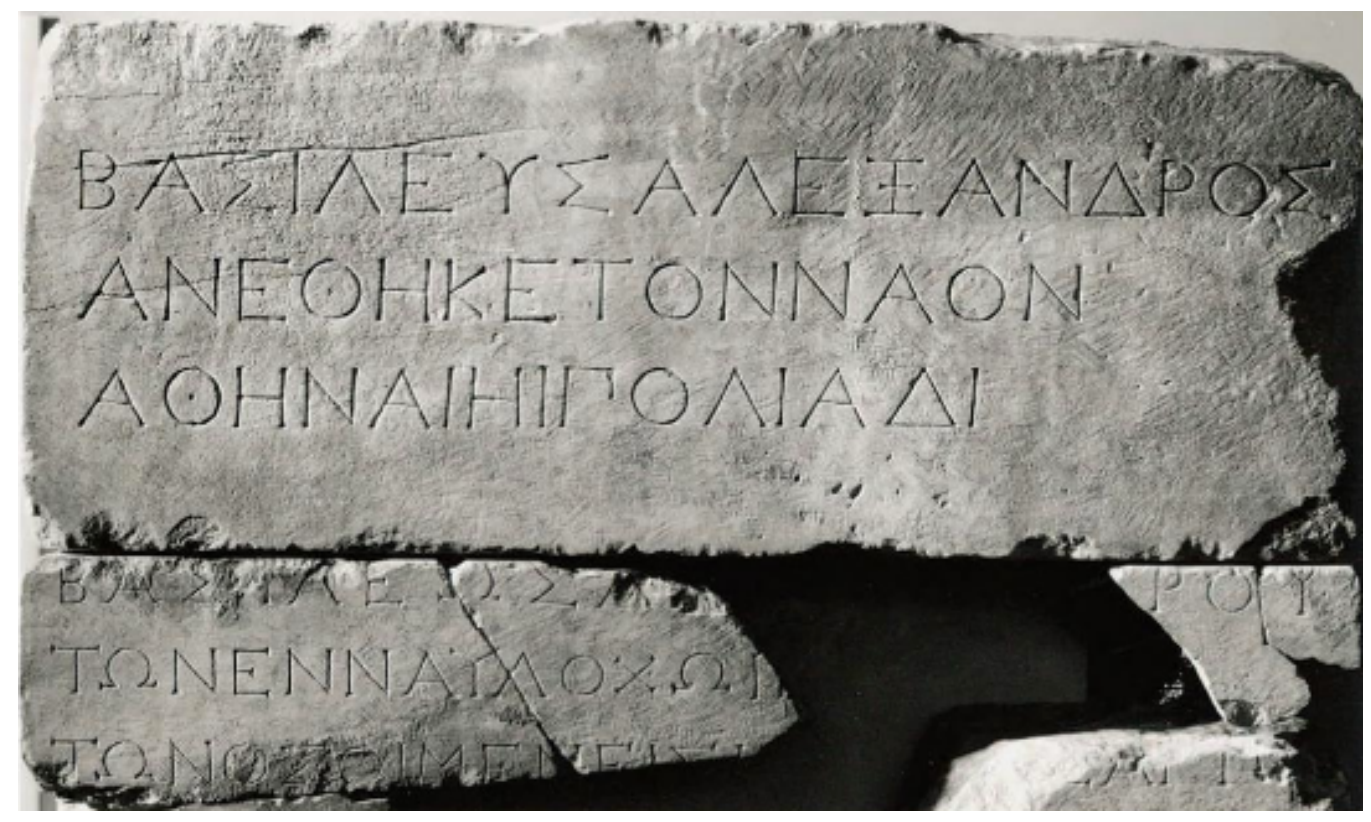

Figure 1: Ancient Greek writing (Poulin, 2012: 28)

Pictographs and rock paintings were the underlying foundation that allowed early civilizations to start communicating along with the development of the ancient Greek writing (see figure 1) (Poulin, 2012). These new forms of communication also allowed early civilizations to construct primary Wayfinding navigation systems that later, would be transformed into vital traditional navigation systems which would be used throughout the world for many years to come. Some of these traditional Wayfinding navigation systems include: signs, symbols and maps. 

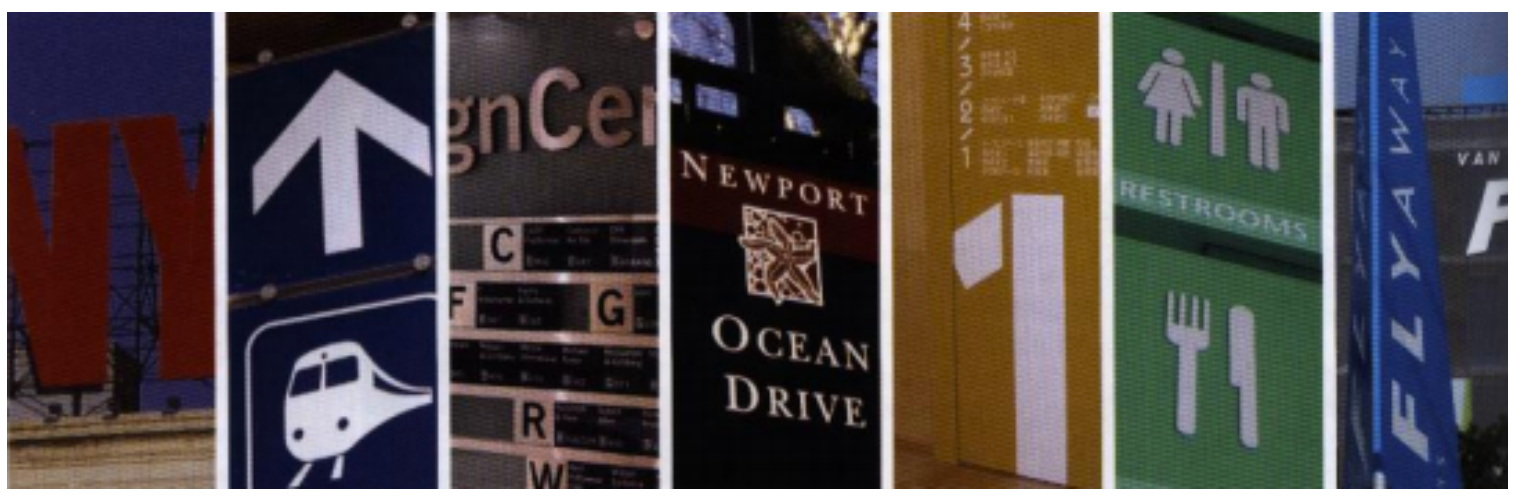

Figure 2: Categories of Signs (Gibson, 2009: 48)

Originally, signs and symbols were created to represent or identify an object. In traditional Wayfinding navigation systems there are four distinct categories in which signs and symbols allow people to navigate from point $A$ to $B$ on an everyday basis. The four categories are Identification, Directional, Orientation and Regulatory (see figure 2) (Gibson, 2009). There are many distinct purposes that these categories serve; however, there are some clear and known examples of these Wayfinding sign and symbol navigation systems. An Identification sign or symbol is referred to: "visual markers that display the name and function of a place or space, whether it is a room, and individual building, or a campus gateway. They appear at the beginning and end of routes and indicate entrances and exits to primary and secondary destinations" (Gibson, 48). These signs are often utilized in spaces such as hospitals, subway stations, airports and shopping malls. A Directional sign or symbol can "constitute the circulatory 
system of a Wayfinding program because they provide the necessary cues that users need to keep on the move once they have entered a space" (Gibson, 2009: 50). Directional signs and symbols can be seen on highways, roads and indoor commercial spaces such as shopping malls.

Traditional sign and symbols used in Wayfinding navigation systems are still commonly seen and utilized everywhere in the world. However, mapping Wayfinding navigation systems are also one of the oldest forms of traditional Wayfinding (Gibson, 2009). In many cases, mapping Wayfinding navigation systems commonly connect and unite signs and symbols in Wayfinding systems (Gibson, 2009).

Routes from mapping Wayfinding navigation systems allow individuals to get from point A to B straightforwardly. Many individuals often know the route that they need to take in order to get to their desired destination in their daily routine. However, a major distinction must be made between Wayfinding in unfamiliar settings and Wayfinding in familiar settings or along familiar routes. On familiar routes, people know what to do to get to their destination. In other words, they have a record of the required decisions and, thus, do 
not need to make decisions-only execute them. Executing decisions is a less conscious and more automatic process than actual decision making, which accounts for the ease with which people follow well-known routes. They retain decisions plans in one form or another and remember or recognize the places where they have to execute specific decisions (Jacobson, 2000: 89).

Although, in unknown spaces, it is important to create clear and universal mapping Wayfinding navigation systems to allow a person to get from one destination to another without any major hesitation. Settings that occupy poor Wayfinding information regularly lead to exploratory decisions and the need to discover relevant information (Jacobson, 2000). Without clear Wayfinding mapping navigation systems, an individual in an unfamiliar space, is required to make uncertain decisions that can lead to undefined areas or spaces.

\subsection{Wayfinding digital transitions}

Systems such as signs, symbols and maps have not only pioneered Wayfinding, but also have allowed designers to pick up fundamental characteristics of these systems and mold them into more efficient Wayfinding technologies in the new digital era. The digital era showcases a transition in the way content is delivered. 
Although various tools in digital media have changed the way content is shown, content itself is still stable. However, new modes of delivery such as social media have also changed the modes of communicating (Grant \& Meadows, 1996). The digital era not only has changed the ways in which we communicate information to one another but also, the way information is communicated through new forms of technologies. We are living and navigating through hybrid environments that connect the physical and digital world. (Jones ,2010). We live in a world where all our surroundings are now being inhabited and connected to digital technologies. Therefore, it is no surprise that Wayfinding navigation systems have transitioned significantly to new and enhanced digital technologies.

\subsection{Wayfinding digital technologies such as GPS and kiosk screens.}

Innovative digital Wayfinding still requires straightforward and well-defined navigation however, the forms of communicating this information has changed drastically with new state-of-the-art technologies such as Global Positioning System (GPS) and interactive kiosks. 
GPS is a digital technology that has transformed Wayfinding. In today's world, any individual travelling to an unknown or known destination uses GPS to find the best and most convenient route. It is interesting to consider that the GPS is less than two decades old. However, by May 2012, half of all American adults$46 \%$ to be exact-owned a GPS-enabled smartphone. Almost three-quarters of them (74\%) access location-based information on phones, such as directions, restaurant and social locations (Farman, 2013).

In many cases, GPS is a technology that provides a link and or connection between maps and position tracking. As mentioned in section 1.2 Traditional Wayfinding methods and systems, physical maps were always used to find a destination through known physical landmarks and characteristics such as signs and symbols (Jacobson, 2000). Position tracking on the other hand, calculates readings from a transmitter and receiver to determine a person's exact location regardless of their surroundings and known physical landmarks (Farman, 2013). However, GSP now, combines mapping systems with position tracking. GPS merges both these systems to not only locate a person on a device but also, to use their location and physical surroundings to determine where they need to go and what routes they should take. The GPS technology has become so dominant and widespread that it is hard to imagine a world without individuals 
using the technology to get from point A to B (Farman, 2013).

Although the technology of the GPS is hard to dissect, the technology can be sufficiently explained by the following: GPS is a network of satellites transmitting radio-signals that can be picked up from almost anywhere on the planet. $\mathrm{A}$ receiver that can pick up time-signals from at least four of the satellites can use time-offsets and triangulation to calculate it's location on the surface of the globe. (see figure 3) (El-Rabbany, 2002). The complexity of the GPS is hidden from mobile-device users, who are presented with an easy and convenient interface.

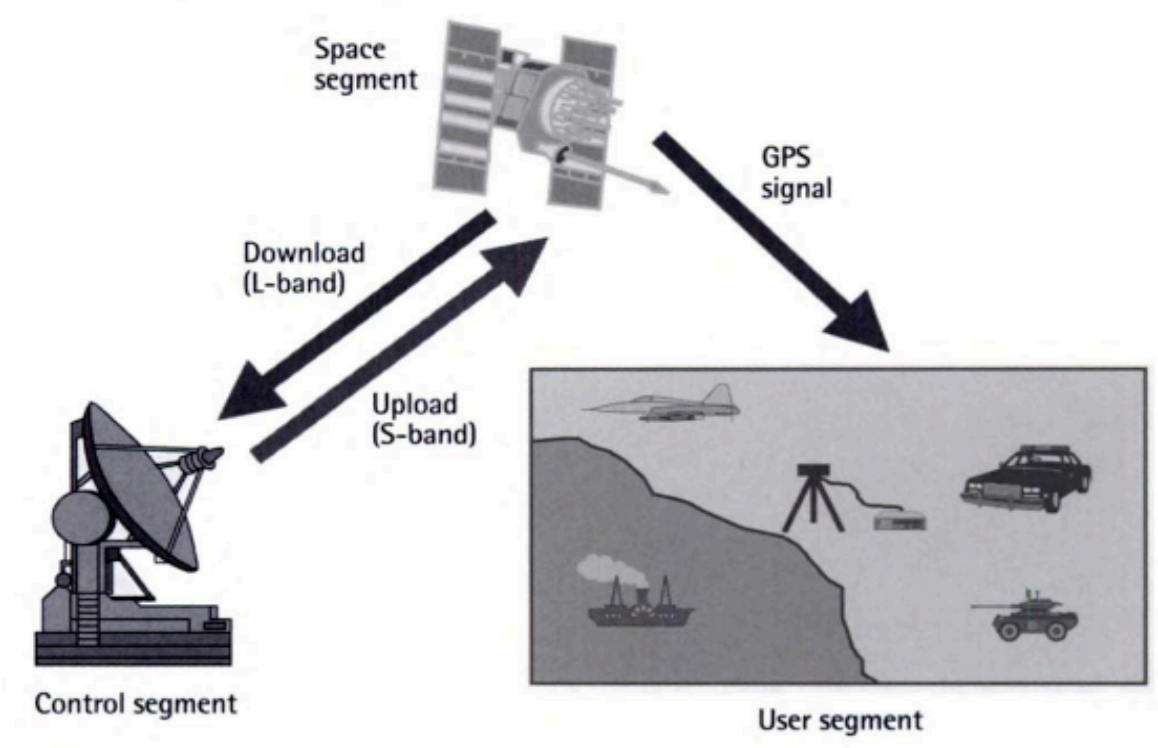

Figure 3: GPS Segments (El-Rabbany, 8) 
The GPS is not the only technology that has transformed digital Wayfinding.

New technologies such as interactive kiosks have also changed the way in which people find their desired destination in popular outdoor and indoor spaces:

commercial or residential. In many instances, these interactive kiosks not only provide improved Wayfinding but also, additional customized features to improve the overall experience for a travelling individual. A perfect example can be found in the Munich airport located in Germany. The InfoGate Information System is a company, which has developed a digital interactive kiosk to provide not only efficient information services but also, enhanced indoor navigation (see figure 4) (Powell, 2015).

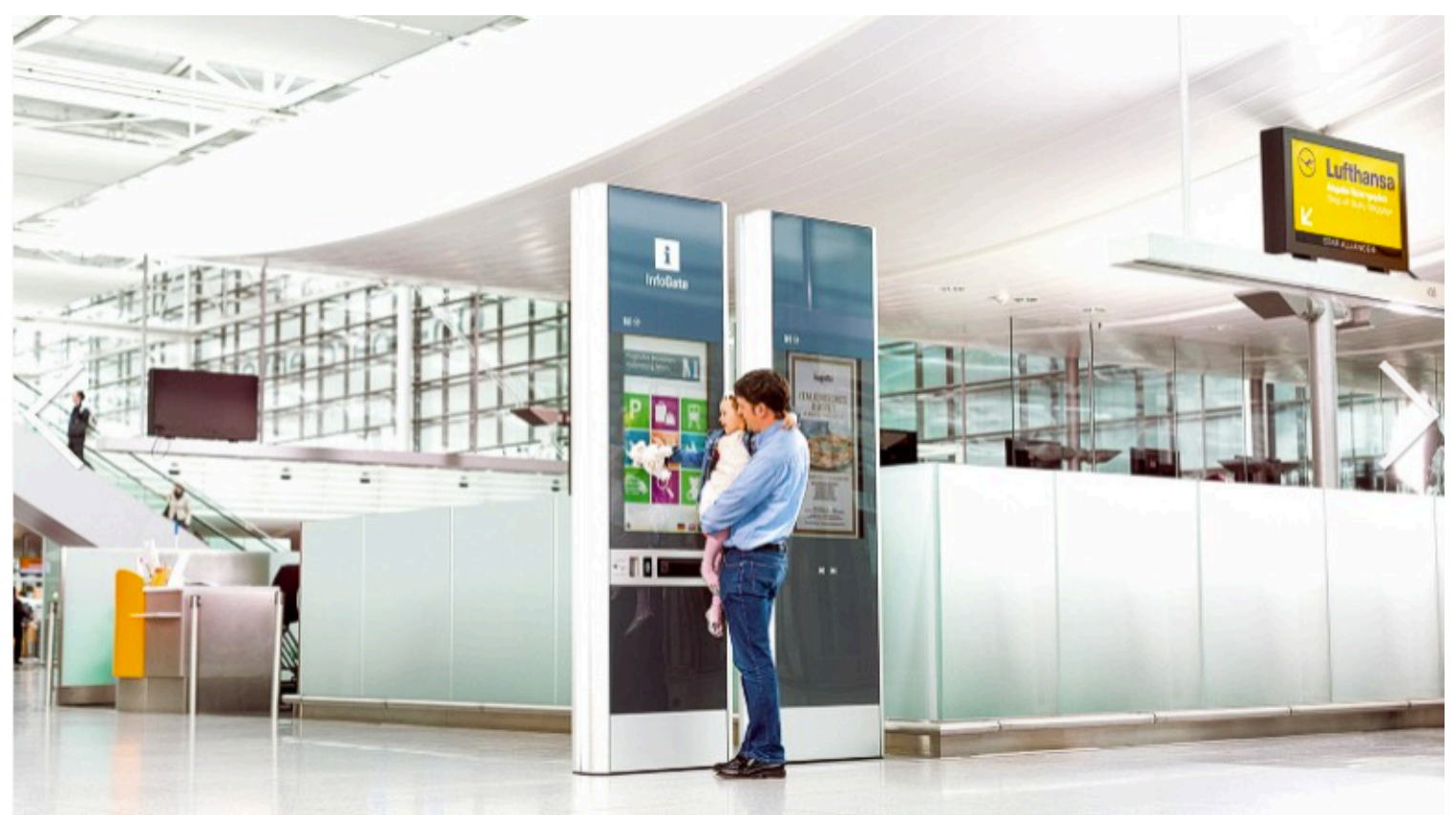

Figure 4: InfoGate Kiosk (Powell, 2015) 
However, it is important to acknowledge that InfoGate's digital interactive kiosks are in fact, evolved versions of traditional map kiosks. Traditional map kiosks use static symbols, signs and maps to showcase information to a traveller or pedestrian. A traveller using a traditional map kiosk sees an overview of their surroundings and is given an indication on where they are in terms of where the map kiosk is located (Gibson, 2009). If a traveller wants to get to a desired destination using a traditional map kiosk, they would have to determine the best possible a route themselves and would not be able to ask any questions if uncertain of their surroundings. This is difficult however; because many travellers have trouble understanding foreign languages and map systems.

InfoGate's digital interactive kiosks allow face-to-face conversation with a live customer representative who speaks the traveller's language. Also, documents can be scanned, printed and exchanged between the representative and traveller. Travellers can get directions by tapping a "you are here" display that gives approximate walking times to their desired destination (Powell, 2015). The InfoGate interactive kiosks conquer the problem of understanding a foreign language very well. In many instances, digital Wayfinding technologies are often used in foreign spaces where language communication is constantly a battle. However, using the interactive kiosks by InfoGate, a person can choose a 
familiar language that can provide information in a clear and straightforward manner.

These innovative digital Way-finding technologies have not only improved Wayfinding, but have allowed designers to understand and implement additional important features and needs for people in complex spaces such as hospitals. 


\section{Chapter Two - Robotic Technologies}

\subsection{Introduction to robotics}

Developments in technology have allowed designers and engineers to create new and innovating technologies that at one point could only be imagined as a fictional concept. It was hard to visualize these technologies to be made in reality. Robotic technologies were once seen as one of those new and innovating technologies.

The term "robot" was first coined in the play called "Rossum's Universal Robots" by Karel Capek in 1920. Capek envisioned a dreamlike scenario with a bioprocess could create human-like machines, devoid of emotions and soul, who were strong and obeyed their masters (Niku, 2010). Although characteristics of the term robot were seen in previous fictional works, the play "Rossum's Universal Robots" showcased a refined definition of what would be known as the modern day robot. Capek's play "Rossum's Universal Robots" only allowed other creatives to produce more fictional works that embodied the characteristics of the modern day robot, which included movies like "Flash 
Gordon, Metropolis, Lost in Space, The Day The Earth Stood Still, and The Forbidden Planet" (Niku, 2010: 4).

These fictional plays and films continuously portrayed an ideal representation of what a robot could look like and function as. However, these fictional productions only sparked an interest in producing actual robotic technologies that could take many forms, serve numerous purposes and be constructed through various disciplines (see figure 5).
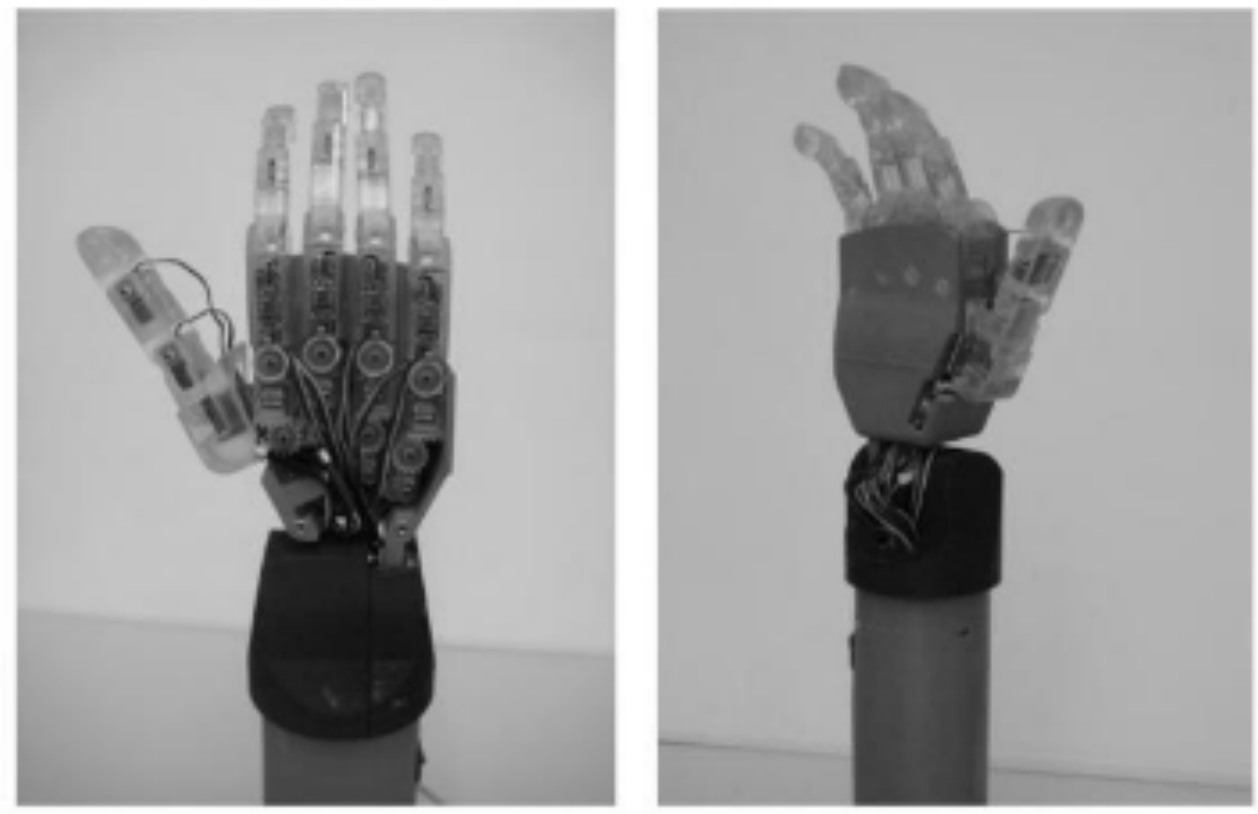

Figure 5: Finger-spelling hand for communication (Niku, 24)

Robotics can be defined as 
the art, knowledge base, and the know-how of designing, applying, and using robots in human endeavors. Robotic systems consist of not just robots, but also other devices and systems used together with the robots. Robots may be used in manufacturing environments, in underwater and space exploration, for aiding the disabled, or even for fun. In any capacity, robots can be useful, but they need to be programmed and controlled. Robotics is an interdisciplinary subject that benefits from mechanical engineering, electrical and electronic engineering, computer science, cognitive sciences, biology, and many other disciplines (Niku, 2010: 4).

Robotic technologies are often difficult to specifically define because, robotics are complex technologies and are often associated with many disciplines.

From the very beginning, robots were always associated with science fiction. No one ever imagined that fictional robotic representations could possibly be produced in the physical world to serve several different purposes. However, new innovative robotic technologies have also, provided superior Wayfinding systems, particularly in health care facilities such as hospitals. 


\subsection{Technological advancements and examples of significant robotic advancements}

Robotic technologies have certainly helped enhance Wayfinding. However, it is important to acknowledge the significant advancements in technology that have assisted in the development of modern day robotic technologies. Also, it is vital to look at early significant robotic advancements to get a better understanding of present robotic technologies with superior Wayfinding systems implemented in hospitals.

Robotic technologies are complex and it is easy to understand how a person can be conflicted on what is and what is not related to the development of robotics. The development of robotics is tied to many other technological advances throughout history. In many cases, various technological advances seem so trivial that people often don't see any relation to the field of robotics. The work of Archimedes, a Greek engineer and inventor, influenced many mechanical devices that are found in modern day robots. Also, Count Alessandro Volta developed the first electrical cell, which provided the basis for portable electric power for many mobile robots. (Angelo, 2007). 
Many past developments in technology as mentioned above, are often associated with the development of robotics. These technological developments however, are consistently disconnected with modern day robotic technologies because many do not see how these developments could possibly be connected to such superior and advanced robotic technologies.

Throughout history there have been several key examples of significant robotic advancements. These advancements have also allowed further research and exploration in present and future possible robotic technologies.

Many of the key advancements in robotic technologies can be seen in space exploration. Robotics in many occurrences, have revolutionized the way in which we see and understand the universe. Since the beginning of the space age in 1957, engineers and scientists have constructed robot spacecraft in various forms. Each space robot was custom designed to meet specific needs and various environmental challenges of a particular space mission. (Angelo, 2007). Designers and engineers in 1957 were already producing customized robotic technologies that could perform specific tasks given to them by their controllers. However, "as space technology matured and was complemented by incredible progress in computer and transitor-based technologies, the complexity of space 
robots changed greatly" (Angelo, 2007:12). Robotic technologies continued to advance and performed more complex procedures in challenging environments.

There have been many operations that have used robotic technologies for space exploration such as Viking 1, Viking 2, Mars Pathfinder and rover later renamed Sojourner, Spirit and Opportunity (Angelo, 2007). These space robots have unquestionably enhanced mobility and various mechanisms that are now used in modern day hospital robotics.

Although the robots mentioned above have allowed scientists to make groundbreaking discoveries in space, the capabilities by these robots were limited by the control and guidance of their operators. As years passed, new robots were constructed to obtain a higher degree of intelligence and independence from controllers.

In 2000, the Honda Motor Company debuted its ASIMO (Advance Step In Innovative Mobility) humanoid robot (Angelo, 2007). This advancement in robotics allowed designers to create a robot that not only could perform complex tasks, but also co-exist with humans with no remote control. 
However, the process of enhancing the ASIMO humanoid robot continued throughout the next few years. Ultimately, on December 13, 2015, Honda introduced the newest form of the company's "people-friendly" humanoid robot. This extended process improved the overall functionality and complexity of tasks the humanoid robot was capable of performing. Also, the humanoid robot acquired more human-like qualities (Angelo, 2007). These enhanced human-like qualities were an important improvement for Honda to make, because the humanoid robot was originally constructed to work and operate in an environment alongside people. In order to complete this fulfillment, the humanoid robot cannot only have physical human capabilities, but also needs to possess a certain degree of intelligence and human sensibility.

The ASIMO humanoid robot was a fundamental technological advancement not only for robotics but also for Wayfinding in hospitals. Technological advancements such as the ASIMO humanoid robot, has allowed designers to create robotic technologies for hospitals, that incorporate superior Wayfinding systems that, which in fact, can interact with their surroundings. However, before examples of these new innovative robotic technologies that have superior Wayfinding systems are stated, it is vital to look at and understand some of the common fundamental components within robotic technologies. 


\subsection{Common components of robotics}

As explained in the previous section, robotic technologies have made huge advancements over the past few years. However, robotic technologies are indeed complex and require an understanding of some of the common underlying components in order to comprehend how these technologies actually work. Robotic technologies serve different purposes and are placed in different settings; however, there are six common components that are utilized in almost every robot.

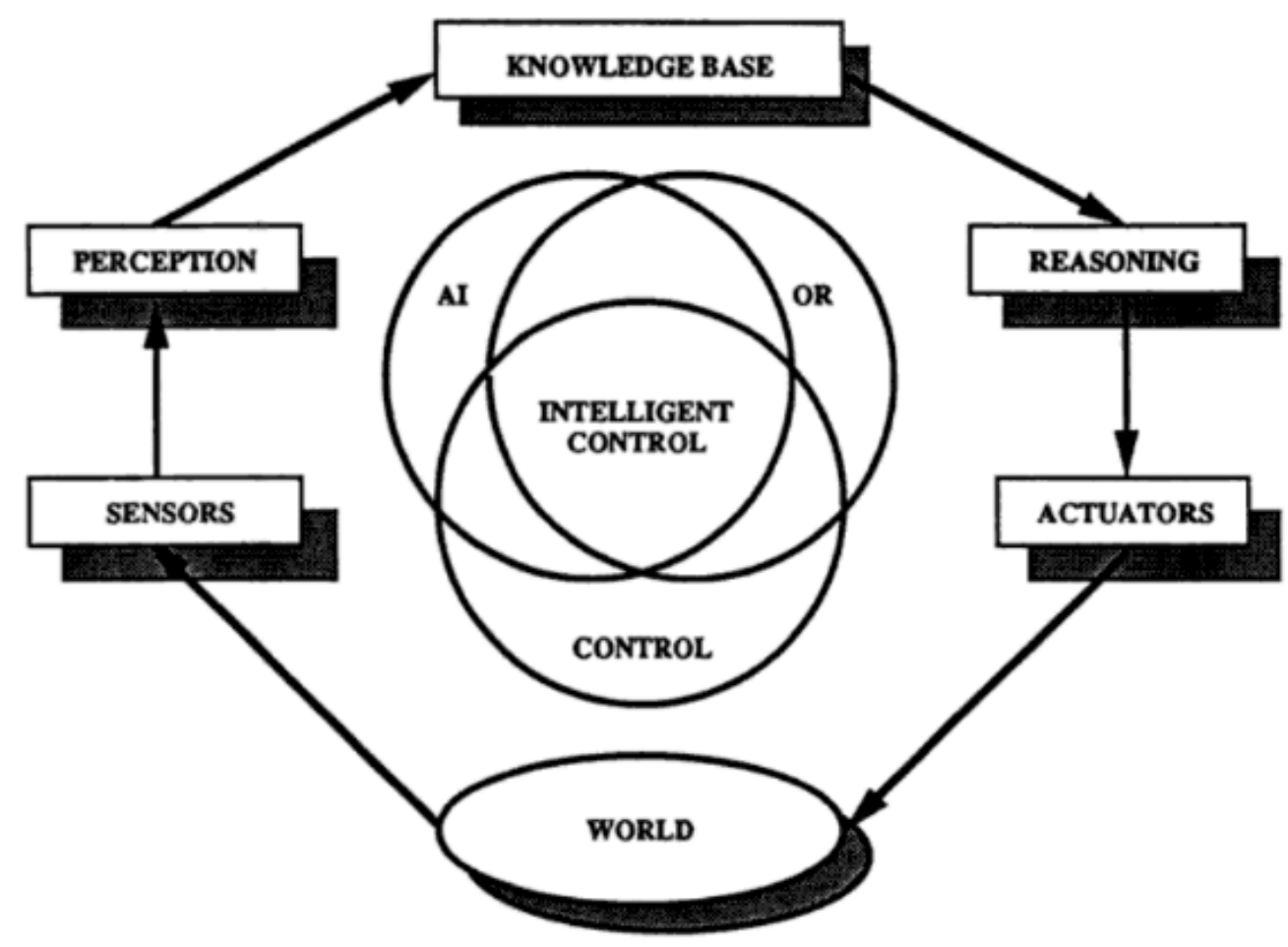

Figure 6: Architecture for Intelligent Control (Caldwell, Gary, 1996) 
The first component is software. In a robot there are three groups of software programs that are used: the first is the operating system that operates the robot, the second calculates the motions of each joint and the third is the collection of application-oriented programs (Niku, 2010).

The second component is sensors. Sensors collect information about the internal state of the robot. Also, sensors are used to communicate with the surrounding environment that includes: a vision system, touch, tactile sensors and speech synthesizer (Niku, 2010). Sensors are the underlying components that allow a robot to "make sense" of the various elements connected within the technology or elements that interact with the robots (see figure 6).

The third component is the processor. The processor calculates the motions of the robots joints that allows a robot to know how fast and how much each joint must move to travel to a location or complete a task. The processor also oversees the controller and the sensors of the robot (Niku, 2010). 
The fourth component is the actuator. "Actuators are the "muscles" of the manipulators. The controller sends signals to the actuators, which, in turn, move the robot joints and links" (Niku, 2010: 7).

The fifth component is the controller. The controller controls the robots motions. For a robot to perform a task accurately and properly, it needs to receive clear communication signals from the controller on what movements to make. The actuator or end effector is dependent on the controller to make the necessary connections to perform various tasks (Niku, 2010).

The sixth and final component is the end effector. The end effector is connected to the last joint of the manipulator and handles objects and performs tasks (see figure 7). The end effector often varies in shape and size, because each robot is constructed to perform a unique task (Niku, 2010).

These six components are found in many robots we see and use in the world today. 


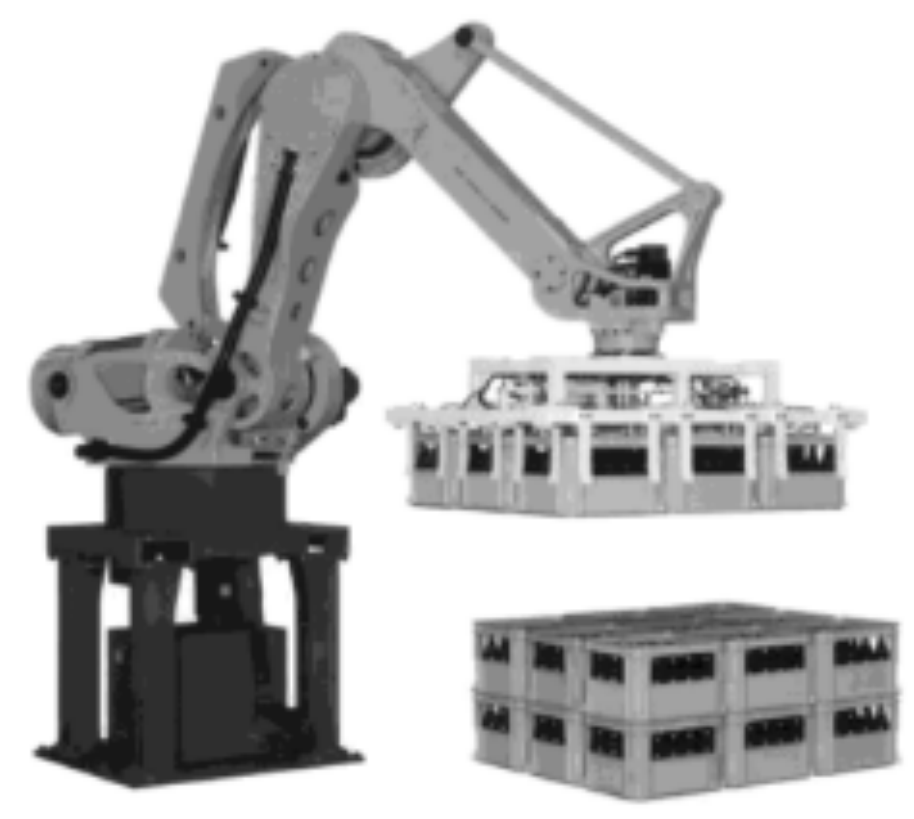

Figure 7: A Fanuc M-410iWW palletizing robotic manipulator with its end effector (Niku, 2010: 7)

\subsection{Robotic technologies with Wayfinding for hospitals}

In the past few years, hospitals increasingly have been incorporating robotic technologies throughout various units. Some of these robots have assisted in complicated surgeries and or diagnoses supervised by doctors and surgeons. However, robotic advancements and understanding robotic technologies have also allowed designers to create enhanced robotic technologies in hospitals that incorporate superior Wayfinding systems to perform and carry out many procedures and tasks. Originally, healthcare workers, nurses and doctors 
performed these tasks and procedures. Not only do these enhanced robotic technologies perform tasks and procedures, they also obtain important confidential information from patients such as medical prescriptions. Additionally, these enhanced robotic technologies have the ability to interact with their surroundings.

Recently, major firms like Aethon in Pittsburgh, have created robotic technologies for hospitals like "TUG", which carry out various tasks and procedures in hospitals (see figure 8) (O'Connor). A TUG uses smart autonomous navigation. A map of a hospital facility is created by Author's implementation team using a highly accurate laser floor dimensioning tool. The facility layout is then programmed with routes that include elevators, autoopening doors, delivery points and TUGs charging stations. The programmed map is loaded into the TUG's memory. The TUG uses the on-board map for guidance and calculates its location in real time through an Aethon-patented odometry algorithm. It uses its on-board sensors to adjust to the changing hallways in real time to safely navigate around people and objects while staying on track with the built in map (How It Works, 2016). Additionally, the TUG uses the hospitals already existing Wi-Fi system to connect with elevators, automatic 
doors and fire alarms. The Tug also uses the Wi-Fi to allow the cloud command center to monitor the TUG 24/7 (How It Works, 2016).

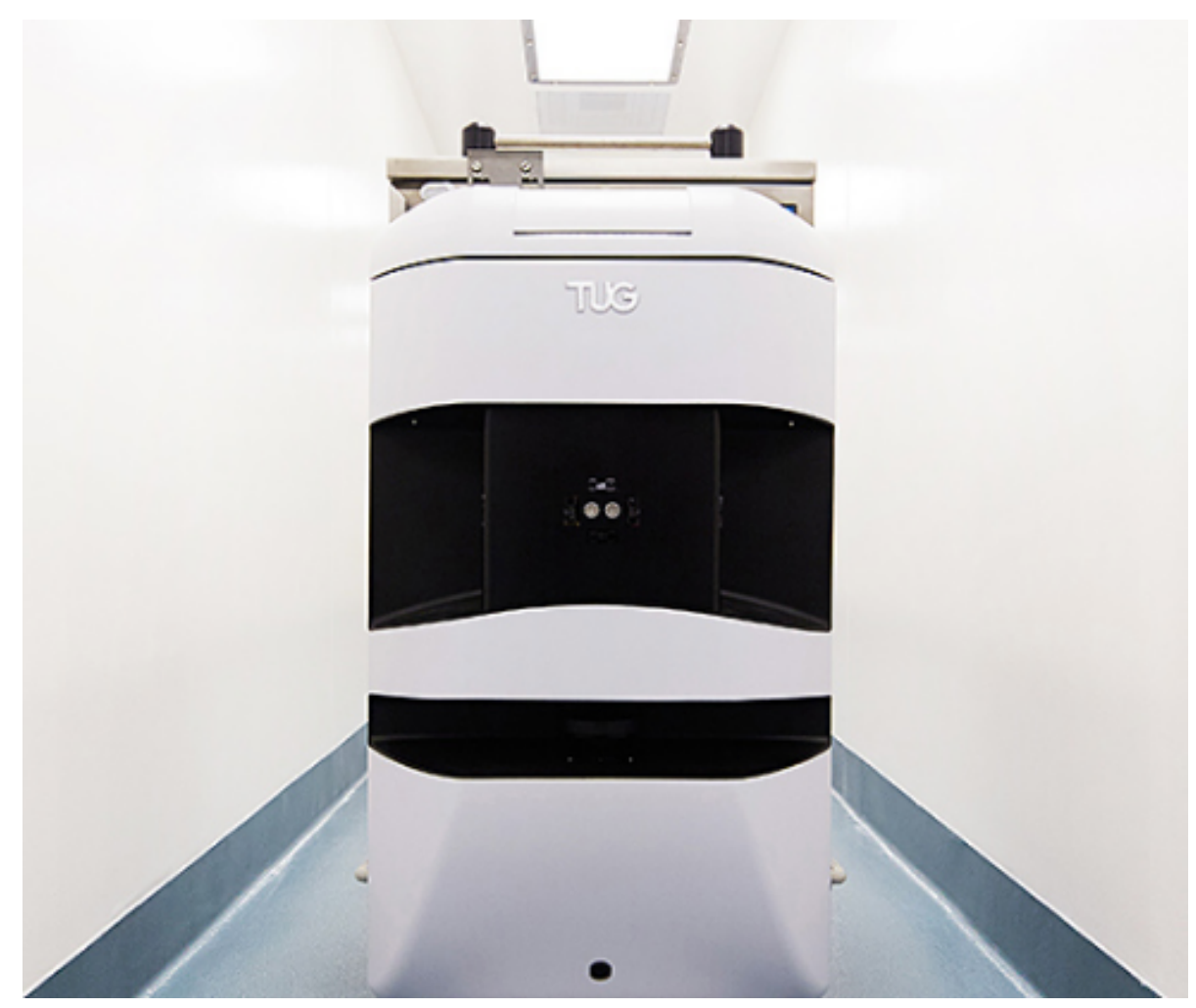

Figure 8: A TUG (High-Tech 'TUG' Robots Will Do Heavy Lifting at Mission Bay, 2016)

Similar robotic technologies like TUG have also, been utilized in the Humber River hospital, which is considered North America's first "fully digital" medical facility (see figure 9) (Davidson, 2015). Just as the TUG, these automated robots assist healthcare workers and nurses by performing various tasks throughout the hospital and bringing food for patients using built in maps, sensors and the hospitals Wi-Fi system (The First Robot Operated Hospital in North America Has Finally Opened, 2015). 


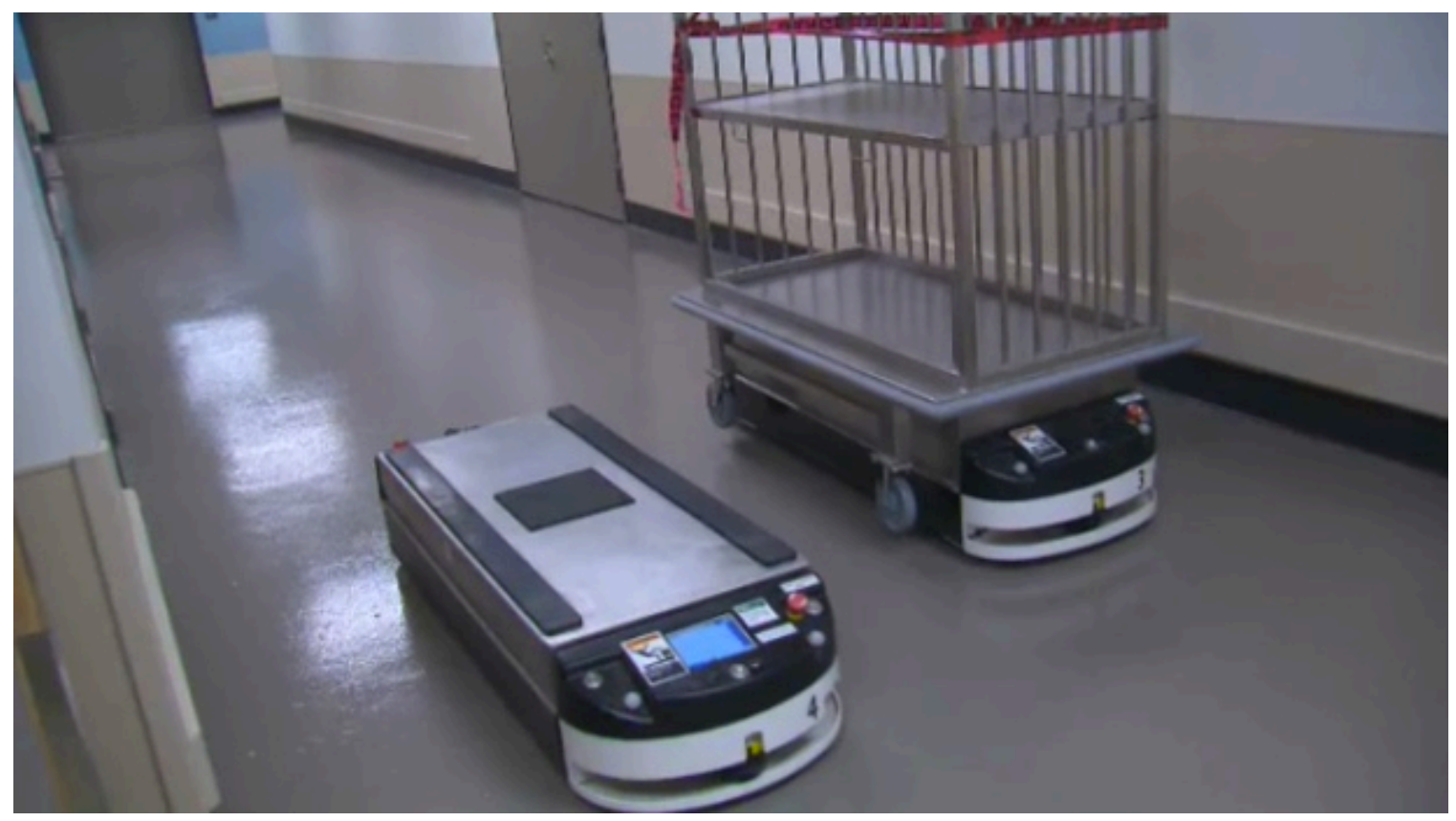

Figure 9: Automated robots at Humber River Hospital (Davidson, 2015)

However, now six out of twenty-four hospitals that are using TUGs are testing an RFID enabled robot dubbed Homer (see figure 10). Homer performs the same jobs as TUG, but also carries an RFID interrogator or reader that is used to locate RFID tagged assets throughout the hospital. (O'Connor). 


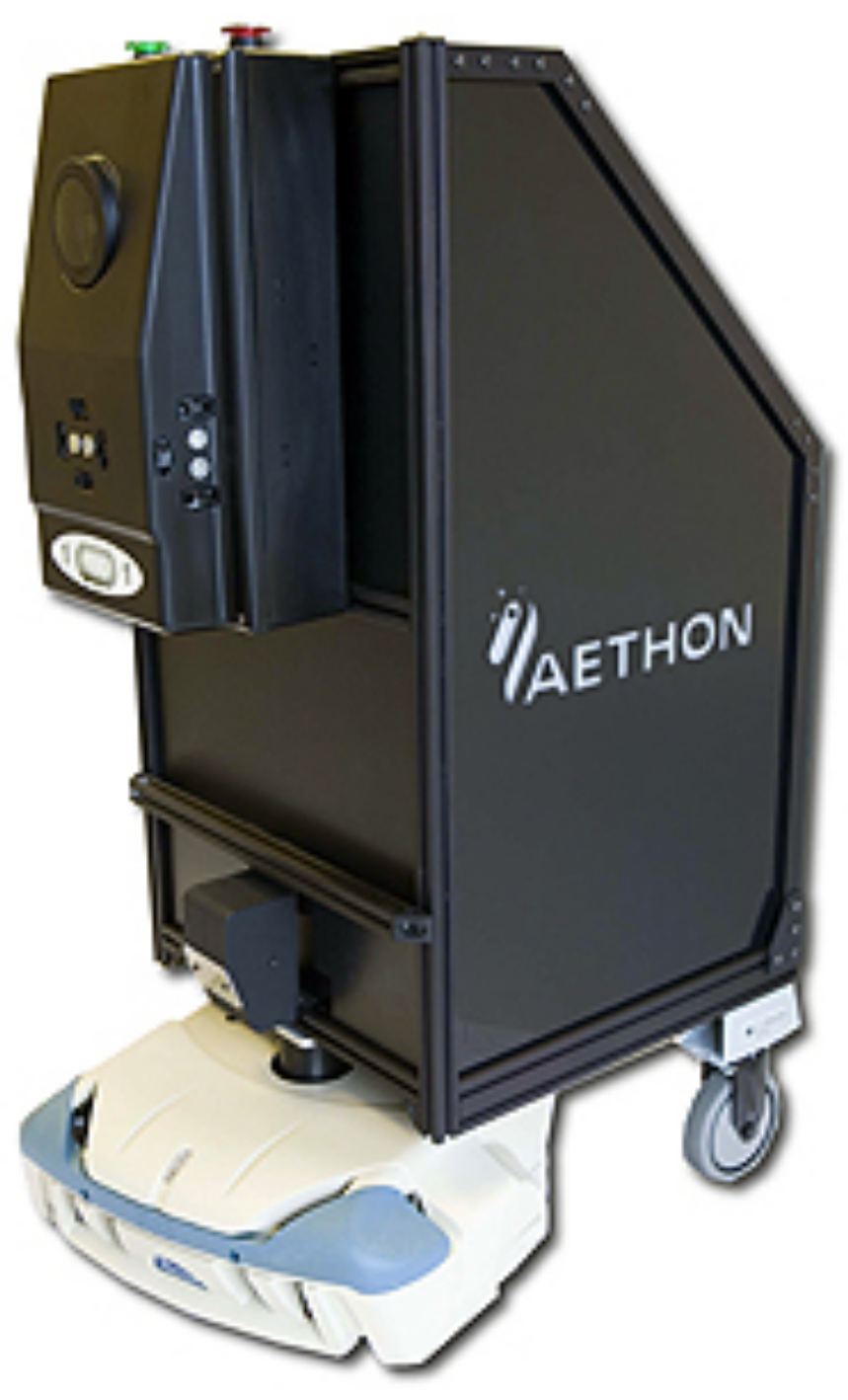

Figure 10: Homer, an RFID-enabled version of Aethon's Tug robot (O'Connor)

Aethon's asset-location software that runs on the automations computer determines the location of each tag, which is sensed by its onboard RFID interrogator. It does this by using a proprietary algorithm to calculate the time it takes for a tags RF signal travelling to reach the readers antenna, while establishing the interrogator's location based on the robots internal mapping function. The software pulls the signal data from the reader when the machine or 
robot is three different distances from the tag. One reading can be conducted at 20 feet away, then 10 feet and the last reading 2 feet away from the tag. The software can determine the tag's location to an accuracy of approximately 3 feet (O'Connor).

Many systems that are in use or implemented in hospitals today which use RFID require a installation of expensive infrastructure of antennas and receivers to find the location of RFID-tagged assets (Havenstein, 2007). However, Aethon's system uses the robots (Homer's) single antenna to locate, deliver and recover assets using RFID tags, and does not require any other sort of equipment to be installed in the hospital (Havenstein, 2007). Therefore, Aethon's system allows Homer to be a more cost effective RFID Wayfinding robotic solution in a hospital.

RFID, RFID tags, programmed maps, sensors and Wi-Fi, have improved Wayfinding in hospital robots tremendously. Not only do these robots have the ability to move throughout the hospital, but they also, interact with their surroundings. In the section "Common components of robotics" sensors and software: that store programmed maps of the hospital have already been described and explained. However, these robots also use RFID, RFID tags and 
Wi-Fi for Wayfinding, which require an understanding of how these technologies actually work.

\subsection{The technology of RFID, RFID tags and Wi-Fi}

In order to understand how RFID, RFID tags and Wi-Fi allow robots to maneuver and navigate their way around the hospital, it is important to understand the technology behind RFID, RFID tags and Wi-Fi.

The RFID (Radio Frequency Identification) technology has progressively been associated with Wayfinding systems in in-door environments. RFID in Wayfinding is commonly considered a more flexible and inexpensive method for determining a pedestrians location and heading with readily accessible information about the building environment, guiding pedestrians along routes, free exploration, and describing points of interest to the pedestrian (Samani et al, 2016). However, in the past few years, designers and engineers have been incorporating RFID in many hospitals to enhance Wayfinding in robots. The main purpose of using RFID technologies in robots is "that the robot can imitate the human behavior using signals of the environment" (Corrales et al, 2016). However, in order for robots to successfully move and navigate their way around 
hospitals, the robots must acquire not only RFID but also RFID tags (see figure

11). RFID tags are signals for a navigation system are placed throughout the environment and are accessible for the robot during navigation. When the robot is moving throughout the hospital to find its desired destination, it explores the environment looking for these RFID tags or signals. Once a robot finds a RFID tag, it reads it, and then analyzes the data to execute the next move (Corrales et al, 2016).

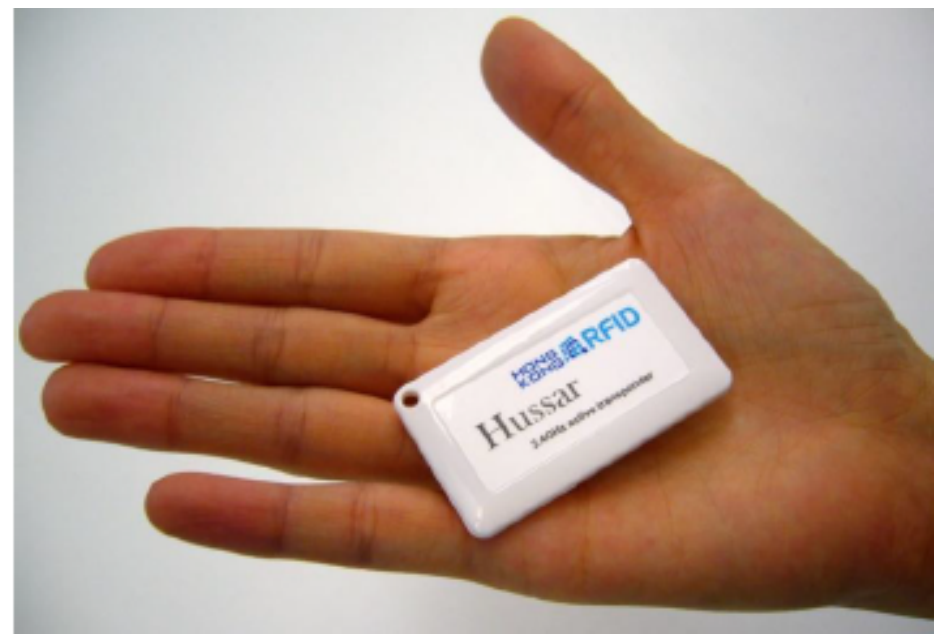

Figure 11: Active RFID tag (Puffer, B.T., 2016)

The RFID tags are a unique piece of technology that allows the robot to pick up signals throughout the hospital environment. However, in order to understand how this is done, it is important to look at the underlying components of an RFID tag. An RFID tag contains a small circuit chip and antenna that is placed within a protective shell. The reader contains at least an antenna and scanner 
that allows communication with the RFID tags. There are two types of tags: battery and passive, which are powered by the reader through radio waves. These tags can also be categorized by working ranges such as near-field and higher band RFID systems. RFID tags can be read-only or read-and-write. The volume capacity of the tags built in memory varies from a few bits to thousands. (Samani et al, 2016).

Wi-Fi is an abbreviation for the term Wireless Fidelity. Wi-Fi is commonly used in many places such as homes, work environments, libraries, universities, airports, shopping malls and restaurants/cafes, that allow you to connect to the Internet at any time (What is Wifi and How Does it Work, 2016). "Like mobile phones, a Wi-Fi network makes use of radio waves to transmit information across a network" (What is Wifi and How Does it Work, 2016).

In Wayfinding, many people commonly rely on GPS and GPS enabled devices to find their desired destination with the best possible routes. However, GPS and GPS enabled devices provide location information from a globally agreed-upon system via satellites that is primarily available for outdoor environments not indoor environments (Gallo). Wi-Fi location however, is a network-based service, which means that any indoor environment utilizing Wi-Fi can place APs (access 
points) throughout a complex and track the location of a person or object without a reference point to base a calculation as the GPS (Gallo).

Implementing Wi-Fi in hospital robots not only allows robots to use indoorpositioning, but also allows the hospital to collect, receive and send data/information. This is made possible because robots that are connected to the hospitals Wi-Fi system are also connected to APs (access points) that are located throughout the hospital. Therefore, systems that are attached to the core Wi-Fi network such as Cisco's Mobility Service Engine or Meru's E(z) RF Location manager can collect, receive and send information alongside determining a robots location relative to their APs (Gallo).

The hospitals Wi-Fi system allows the hospital to not only track robots, but also allows the hospital to monitor drugs that are stored throughout the hospital or delivered to patients by robots. Additionally, some healthcare workers who have mobile devices that are connected to the hospitals Wi-Fi can also be tracked and monitored throughout the hospital to ensure patient care (Gallo). 
In many instances, implementing Wi-Fi in hospital robots for Wayfinding is easy and cost effective, because hospitals already use $\mathrm{Wi}-\mathrm{Fi}$, which would require no additional technologies to be set up or placed throughout the hospital.

RFID, RFID tags and Wi-Fi technologies have undeniably improved navigation in hospital robots along with other robotic components. However, these robots that are being implemented in hospitals are raising questions on whether these robots will eventually replace and take the jobs of healthcare workers and nurses and take the humanity out of healthcare. Additionally, if these robots are in fact, safe to operate in hospitals. 


\section{Chapter Three - Ethical and Safety Concerns about Robots in Hospitals}

\subsection{They are just like one of us, apart of our team}

The world's fascination of robots and their capabilities continue to grow every day. Now, in many cases, it is hard to imagine a world where robots never existed.

In recent years, robots such as the da Vinci has been implemented in hospitals

for surgery and diagnoses purposes that are controlled and operated by surgeons and doctors (Ross, 2012). However, now, humanoid robotic technologies such as TUG and Homer are being utilized in many hospitals around the world. Robots like TUG and Homer not only complete important tasks and procedures, but these robots also have the ability to interact with their surroundings.

In many cases, "apart from childbirth, it's hard to think of anything a robot cannot easily replicate" (Brockwell, 2015). As mentioned above, robots such as TUG and Homer acquire many humanistic characteristics, which in fact, allow 
them to do almost anything. In the future this may even include the ability to learn anything, reason, use language and form original ideas (Brockwell, 2015). Envisioning robotic capabilities as mentioned above, has only heightened the interest in implementing more robots in hospitals.

TUG and Homer are robotic technologies; however, these robots do poses humanistic characteristics, which allows healthcare workers, nurses and doctors in hospitals to start treating them as if they were apart of the healthcare team. TUG and Homer play a vital role in hospital settings by completing important tasks and assisting hospital staff with many procedures. Therefore, it is to no surprise that healthcare workers, nurses and doctors are considerably respectful to these robots (Simon, 2015). In many instances, if anyone is trying to get in the way of a robot doing its job, many healthcare workers are often heard saying, "Hey, the robot's got a job to do. Stay out of their way" (Simon, 2015). Interesting isn't it? Healthcare workers are automatically treating the robot as if it was another healthcare worker that was apart of their team.

Healthcare workers, nurses and doctors are also, being coached on how to deal with robots (Simon, 2015). When robots were newly implemented in hospitals, it was to no surprise that it was going to be a challenge to have both humans and 
robots operating in the same setting. Hospitals now, are finding it vital to train hospital staff on robot etiquette (Simon, 2015). In hospitals, trainers are commonly telling the hospital staff to treat robots as if they were their grandmas, and as if they were in the hospital in a wheel chair. If something comes in their way they should move aside and don't stand in front of them (Simon, 2015). Once again, these robots are being given the same importance as other healthcare workers and nurses in the hospital.

It is quite evident that healthcare workers, nurses and doctors now, perceive robots like TUG and Homer as apart of their team. However, "most staffers have a strange nonreciprocal affection" for robots as well (Simon, 2015). This behavior towards robots is not at all surprising. Since TUG and Homer do acquire many human characteristics, it allows healthcare workers, nurses and doctors to start treating these robots like they have actual feelings. In the University of California, San Francisco's Mission Bay wing, a woman wearing scrubs was seen trying to move an object she had placed that was blocking a TUG, as if she had ruined the robots day. After being able to finally move the object, she shoved it aside and said "Go TUG go!" (Simon, 2015). TUG and Homer also have the ability to communicate and speak with the hospital staff. The "TUG is chatty. Lest you worry that it's broken down while waiting for the elevator, its assures 
you: "Waiting for a clear elevator." Once it gets one: "Waiting for doors to open." TUG warns you when it's about to back up, and thanks you after you've unloaded its delivery" (Simon, 2015). The ability for TUG and Homer to communicate and speak has progressively allowed healthcare workers, nurses and doctors to feel like they are having a conversation with a co-worker and or staffer, which in many cases, can ignite some sort of affection and or fondness towards these robots.

Many question why designers and engineers are trying to humanize robots. What is the purpose? What are the benefits? How will it affect society? David Hanson, CTO of Hanson Robotics, states that humans are brilliant, beautiful, compassionate and undeniably intelligent creatures. Therefore, why shouldn't we aspire to make robots that possess the same qualities as humans? Although robots do not completely possess these qualities yet: we should strive to produce robotic technologies that can live up to these standards (Hanson, 2011). This methodology is quite noteworthy for hospital robots because, a hospital is not only a complex but sensitive environment. If robots are placed in hospitals and have the ability to interact with patients, robots ought to acquire humanlike qualities. These qualities will ensure that patients are being treated 
with the same care and friendliness as if they were being taken care of by healthcare workers or nurses.

However, designers and engineers are also humanizing robots to ensure the safety of humans. Robots may deem to be dangerous if they are not humanized and have posses a certain level of intelligence. When robots are 'awaken', they need to attain profound understanding and compassion towards people around them (Hanson, 2011). This will ensure that robots can exist alongside humans instead of posing a threat towards them.

In the past, however, robots completed and performed tasks that were controlled by controllers. These robots did not acquire a high level of intelligence because they did not have to interact with humans. However, robots that are being produced now, are being produced so that they can interact and co-exist with humans.

\subsection{Will these robots replace healthcare workers and nurses? Will these robots take the humanity out of healthcare?}


By possessing humanistic characteristics and capabilities, robots have indeed gained a lot of admiration and respect from healthcare workers, nurses and doctors. However, these characteristics and capabilities that TUG and Homer poses have also, become a threat to healthcare workers, nurses and in some cases, doctors. The tasks and procedures performed by TUG and Homer are constantly being compared to the work and moreover the quality of work that is being conduced by healthcare workers, nurses and on some occasions even doctors. This is rising the question if robots, are in fact, more valuable and efficient than healthcare workers and or nurses?

Hospitals have looked into three factors that have heightened the interest of implementing robots over healthcare workers and nurses: lower costs, reduction of errors and the nature of healthcare workers and nurses compared to robots.

Robots like TUG and Homer "operating for two shifts a day, seven days a week, can perform the work of at least two full-time staff yet cost less than one person, so there is an immediate cost saving" (Ross, 2012). Overall, implementing robots would save the hospital a lot of money. Hospitals would not have to worry about paying a hefty salary to two full time healthcare workers or nurses. 
Instead, the hospital would receive more labor out of one robot compared to two healthcare workers or nurses performing the same daily duties.

The second important factor of implementing robots have risen out of the fear of mistakes and errors that healthcare workers and nurses can make throughout their shift at the hospital (Lee, 2013). Robots are designed to follow orders and commands, and are not side tracked by their surroundings. Once a job is completed and only completed properly, the robot moves on to another task (How It Works, 2016). Hospitals believe that this level of precision and accuracy can only come out of robotic technologies such as TUG and Homer.

The third important factor comes from the very nature of healthcare workers and nurses compared to robotic technologies. Robots like TUG and Homer can be operated at any time and for any length of time as needed by the hospital, without having the concern of being over worked or giving them breaks. In many cases, hospitals are happy with their tireless workers operating in a stressful environment with many tasks and procedures that need to be taken care of (Ross, 2012). The ability to operate robots for countless hours and by not having to treat them with the same principles and moralities as healthcare workers or nurses, have indeed intrigued hospitals to purchase more robots. 
Although these three factors do favor implementing robotic technologies like TUG and Homer over healthcare workers and nurses, many hospitals are stating that robots are actually supplementing current jobs and creating more jobs instead of removing jobs.

While robots are estimated to be cheaper to install in hospitals, an Oxford study calculated that nurses in fact, have less than $1 \%$ chance of having their jobs being replaced by robots or being automated. The Bureau of Labor Statistics also, stated that nursing is one of the fastest growing professions. In fact, by 2022, registered nurses employment will increase by 19\% (Del Prado, 2015). Robots will not be able to replace hospital staff because; robots cannot provide the same care as healthcare workers and nurses (Su, Liu, Lazar).

Though robots do have the capability to carry out tasks and procedures like healthcare workers and nurses, many hospitals are stating that these robots are and should be considered as an addition to the hospital staff. In many cases, implementing robots like TUG and Homer are helping out with the current staff so they "aren't pushing around huge carts, they're not straining themselves or moving down their colleagues" (Simon, 2015). 
Additionally, the ability for TUG and Homer to carry out many tasks and procedures allow healthcare workers and nurses to spend more of their time caring, catering and providing overall better service to their patients.

Surprisingly, robots also assist healthcare workers and nurses to provide improved experiences for their patients. As mentioned previously, The TUG is able to cater to the patients needs by bringing them their needs and wants within a hospital such as food, medicine and other daily needs (The First Robot Operated Hospital in North America Has Finally Opened, 2015). However, a handful of other robots in hospitals also have the ability to enhance patient experience. Various therapy robots provide meaningful emotional connections to patients. Also, telemedicine robots allow a doctor to perform diagnoses even if they are miles away. At first, patients are skeptical of telemedicine robots. However, after a few minutes, patients actually feel that they are having a conversation with their doctor as if they were present in the room (Su, Liu, Lazar).

Hospitals are also reassuring that robots are in fact, creating new jobs. TUG and Homer are robotic technologies that require a team or group of technicians and monitors to look over the activity and performance of these robots (Simon, 2015). These robots are not only assisting healthcare workers and nurses with 
their jobs, but are also allowing the hospital to hire staff to look over robots like TUG and Homer, to ensure that they are doing their job.

Although some hospitals are stating that robots are not taking over healthcare workers and nurse's jobs, It would be inadequately optimistic to say that robots like TUG won't overstep on more jobs as they grow more sophisticated (Simon, 2015). Robots will only grow to be more intelligent and perform even more complex tasks in the future, and as said "artificial intelligence only gets better-it never gets worse" (Brockwell, 2015). Soon, hospitals will question if they even need healthcare workers or nurses in hospitals settings at all. Robots are already being exhibited to be less expensive to operate in hospitals, and many workers doing tedious jobs have already been replaced by robotic technologies in various other workplaces and disciplines (Brockwell, 2015).

In this past few years, smart and sophisticated robotic technologies have drove rapid economic progress for various companies because of lower costs and the ability to perform the same tasks as human. However, the rapid economic growth made by these smart robotic technologies have decreased the amount of economic contributions made by citizens; primarily from the middle class. Additionally, the more impact and significance these robotic technologies have 
in the global economy, the value of human labor will decrease at a bewildering speed (Davidow \& Malone, 2014). An example of this can be seen in the company Foxconn. Foxconn is the world largest contract manufacturing company that employs over one million workers in China. In 2011, Foxconn installed around 10,000 robots that were dubbed Foxbots. Each Foxbot cost the company around $\$ 20,000$. However, in 2013, Foxconn's CEO Terry Gou, announced that the company would be aiming to add one million robotic workers in the future. Adding one million robotic workers would evidentially allow Foxconn to avoid hiring more human workers. (Davidow \& Malone, 2014).

Companies all over the world like Foxconn, are already seeing the value in implementing robots instead of humans for labor. It would not be any surprise if this would be the fate of healthcare workers and nurses in hospitals. As mentioned above, hospitals are already looking for less expensive methods of labor that are being found in robotic technologies. An example of this can be seen in the new Humber River hospital, that has invested 1.8 billion dollars to dub Humber River hospital as "North America's first all-digital" hospital. Inside, there are robots sorting out drugs, automated robots serving patients lunch and robots carrying blood samples to labs. Also, $75 \%$ of all the back-of-the-hospital 
functions such as laundry and pharmacy are already digitized (Kutscher, 2016). In the future, hospitals may be operated entirely by robots.

Hospitals are indeed fascinated and intrigued by robotic technologies and continue to make large investments on incorporating these robotic technologies into hospitals. However, there are questions, which are being raised that hospitals will soon be taking the humanity out of healthcare by bringing in more robotic technologies. Will patients feel comfortable being surrounded only by robots? Will patients feel safe that robotic technologies are taking care of them? And finally, will taking away human contact actually change hospitals for the better?

However, to envision or even consider a hospital without any human contact, it's important to look at how safe and secure robotic technologies like TUG and Homer actually are.

\subsection{How easy is it to hack into these robots and obtain confidential information?}


Hospitals are continuing to purchase robotic technologies. However, apart from looking at the benefits of implementing these robots, it's also vital to comprehend if these robotic technologies are in fact, secure to operate in hospitals. Numerous technologies are constantly being hacked into today, and this also raises security concerns about operating robotic technologies in hospitals.

Robots like TUG and Homer do utilize many sophisticated technologies such as sensors, programmed maps, RFID and RFID tags to maneuver their way around the hospital, and these technologies can in fact, be hacked into (Zetter, 2014). Hackers usually gain access to these technologies by infecting an employee's computer, which is connected to these technologies. Subsequently, hackers explore the internal network to find the most vulnerable technologies. This is made possible because, these systems often lack authentication to enter or manipulate the system or equipment: which include weak passwords that can be easily remembered such as 12345 or admin. Also, embedded web servers and interfaces make it simple to manipulate devices once a hacker can find them on the network (Zetter, 2014). 
However, apart from these technologies, the most vulnerable technology that robots are connected too is the hospitals Wi-Fi. As mentioned in chapter two, the hospitals Wi-Fi system allows robots to connect with automatic doors, elevators and fire alarms. TUG and Homer also use the Wi-Fi system to allow the cloud command center to monitor and store information from these robots 24 hours a day and seven days a week throughout the hospital (How It Works, 2016).

Many security researchers have demonstrated how hackers can hack into a hospital's Wi-Fi systems. There have been many accounts where hackers have been able to hack into a hospitals Wi-Fi system and gain access to almost everything inside: confidential information, patient data and even control over various robotic devices (Snow, 2016). Hacking into a hospitals Wi-Fi system is indeed very easy; a hacker in a hospital can merely plug his or her laptop into the network and attack vulnerable systems. Once a hacker is through into the network, the hacker can scan and find almost any device (Zetter, 2014).

While developers are constantly trying to search for vulnerabilities and infrastructure issues within hospitals Wi-Fi systems, hospitals are still lacking in 
cyber security and still find many hackers successfully hacking into their Wi-Fi systems (Snow, 2016).

Although the hospital's Wi-Fi system is one of the most vulnerable technologies, hospitals need to acquire more about cyber security for all their devices and technologies which include: robots (automated and surgical), Wi-Fi, insulin pumps, defibrillators and etc. Knowledge about cyber security will stop hackers from controlling and manipulating devices and technologies and accessing confidential information. Evidentially, there are two groups of people should be concerned about cyber security in hospitals: developers of medical devices and hospital management boards. Developers of medical devices and technologies should test their products and search for vulnerabilities. Also, developers should perform regular audit and security checks. Management boards should look into their network security and make sure that no critical infrastructure is connected to networks that are also available to the public. Also, hospital management boards should perform security tests on all devices and technologies before distributing them throughout hospital units (Snow, 2016). The lack of cyber security in hospitals is of no surprise. Typically, medical devices and equipment are often inspected for reliability, effectiveness and safety. Hence, security of these devices and technologies is often overlooked (Zetter, 2014). 
Not only are there concerns of third parties hacking into robotic technologies, there are also concerns about trusting robots like TUG and Homer operating in environments where people's lives are constantly on the line.

\subsection{Are robotic technologies like TUG and Homer safe to be operating in a environment where peoples lives on are on the line?}

Although robotic technologies are not new, many people particularly patients, find it difficult to comprehend that a robot can possibly be responsible for carrying out responsibilities that may indeed affect their lives.

Robots like TUG and Homer are not only responsible for taking care of tasks around the hospital, but they are also responsible for delivering and storing medication around the hospital (Bridges, 2015).

A pharmacist gathers some drugs, scans their codes into a touchscreen next to the robots, and chooses the destination for each. Walking over to Wall-E (a name given to one of the TUG robots), she enters a code on a number pad, then places her thumb on a biometric reader to unlock the machine. A small screen on the robot tells her which medication goes in which numbered drawer, and she proceeds to pop each open and place 
the drug inside. With a tap of the green button atop Wall-E, the robot is off (Bridges, 2015).

Although this process may look appealing to handle medication that is being sorted and delivered to patients, it also raises questions if patients actually do feel secure or even trust taking medication from a robot instead of a nurse or healthcare worker. "Imagine lying in your hospital bed, recovering from surgery, only to see a robot glide in, ready to top up your pain meds. You watch as its smooth, silicone-coated hand extends a tiny paper cup holding your pill" (Bridges, 2015). Many questions could possibly be going through a patient's head such as: Where did this medication come from? Is this in fact the right medication? Are these medications safe? However, it is also important to understand that patients may have questions and concerns about their medications and overall health that a robot possibly cannot answer.

The question of trust is a huge factor in hospital robots. Throughout the years, designers and engineers have been trying to build robots for hospitals that encourage not only healthcare workers and nurses but mainly patients to trust them. Previously, robots were being built to complete and perform various tasks without considering any aspect of their surroundings: predominantly humans. However, to build a robot that stimulates trust now, robots need to be built 
based on human behavior, social rules and laws that encourage a robot to do the "right" thing just as a human. Also, roboticists now, are working with psychologists, sociologists, linguists and anthropologists to get a better understanding about what makes us humans trustworthy and reliable. Therefore, this combined process allows roboticists to build robots that acquire humanistic characteristics, which in return, can be trustworthy (Hassler, 2016). As mentioned in chapter two, a TUG has the ability to communicate with humans. Also, the TUG has the ability to store patient information that allows the robot to identify a patient when entering a room (Simon, 2015). Patients are more likely to trust robots if they are able to recognize them and explain their intentions as humans would (Hassler, 2016). Although hospital robots do acquire many humanistic characteristics, the concept of "ethics" in robots is still far-fetched. Inabilities for robots to make ethical decisions in hospitals continue to allow patients to feel a certain degree of uncertainty (Hassler, 2016). After all, robots are not conscious creatures as are humans.

Also, it is important to know that while technicians can track TUGs movements throughout the hospital, it isn't always easy to pinpoint, divert or stop them (Simon, 2015). If a robot accidently has the wrong medication placed in its compartments, it would be more difficult to track them down compared to the 
hospitals staff. This is important to note because, a robot can only follow commands and instructions that are keyed into their systems by healthcare workers and nurses. If the wrong medication is placed in a robots compartment, the robot will not be able to distinguish if the medication is the correct or not. Also, this tracking issue raises the risk of patients possibly in taking the wrong medication, without any clue which can possibly lead to more health issues or problems that can be undetected for a period of time.

In many circumstances, "there's something unsettling about a robot that's responsible for human lives tooling around with minimal commands" (Simon, 2015). While operating a TUG and Homer is in fact easy, the simplicity of these robots raises the most concerns. A hospital setting is complex, and requires robots to understand more complex commands to ensure the overall safety of patients.

This chapter raises many questions about whether or not robots like TUG and Homer with superior Wayfinding technologies should be placed in hospitals. However, advances in robotic technologies and Wayfinding are creating many projections on what the future of hospitals will look like with even more advanced robotics. 


\section{Chapter Four - Future Projections, Concerns and Conclusions}

\subsection{The future of robots in hospitals}

Advances in robotics and Wayfinding have indeed changed hospital settings drastically. Today, many patients are being greeted and being taken care of by robots. Although robots like TUG and Homer have raised many ethical and safety concerns about these robotic technologies operating within hospitals, designers and engineers are constantly exploring the field of hospital robotics to produce even more sophisticated robots than TUG and Homer with superior Wayfinding systems.

Robots like TUG and Homer acquire many humanistic capabilities, that allow these robots to complete various tasks and procedures that originally, would have been taken care of by healthcare workers and nurses ( $\mathrm{O}^{\prime} \mathrm{C}$ onnor). These humanistic capabilities are the very reason why hospitals are increasingly investing in hospital robots. 
However, a true humanoid robot would be a recreation of the human thought process and have our intellectual abilities. The humanoid robot would have the ability to learn just about anything and possibly form original ideas (Brockwell, 2015). Designers and engineers are looking to enhance not only the capabilities of robots, but also their ability to think and reason just like healthcare workers and nurses. These robots "therefore would display a rich diversity of projects where perception, processing and action were embodied in a recognizably anthropomorphic form in order to emulate some subset of the physical, cognitive and social dimensions of the human body and experience" (Brockwell, 2015). If a true humanoid robot (or droid) were in fact created, it would significantly heighten the concern of jobs for healthcare workers and nurses. If a humanoid robot (or droid) has the ability to not only perform tasks, but also to think and reason like a healthcare worker and nurse, what would be the purpose or point in keeping both? Especially when robots in hospitals have indeed proved to be cheaper to operate within hospitals than healthcare workers and nurses. Not only will these projected humanoid robots (or droids) have the ability to act and think like a human, these robots may soon look like humans to (see figure 12). Soon, we will see a robot that is indistinguishable from a human being. There are already unbelievable enhancements that are being made with voice recognition and language. Also, the mechanics of human movement along 
with "human like" skin are also in the process of being conceptualized.

(Brockwell, 2015).

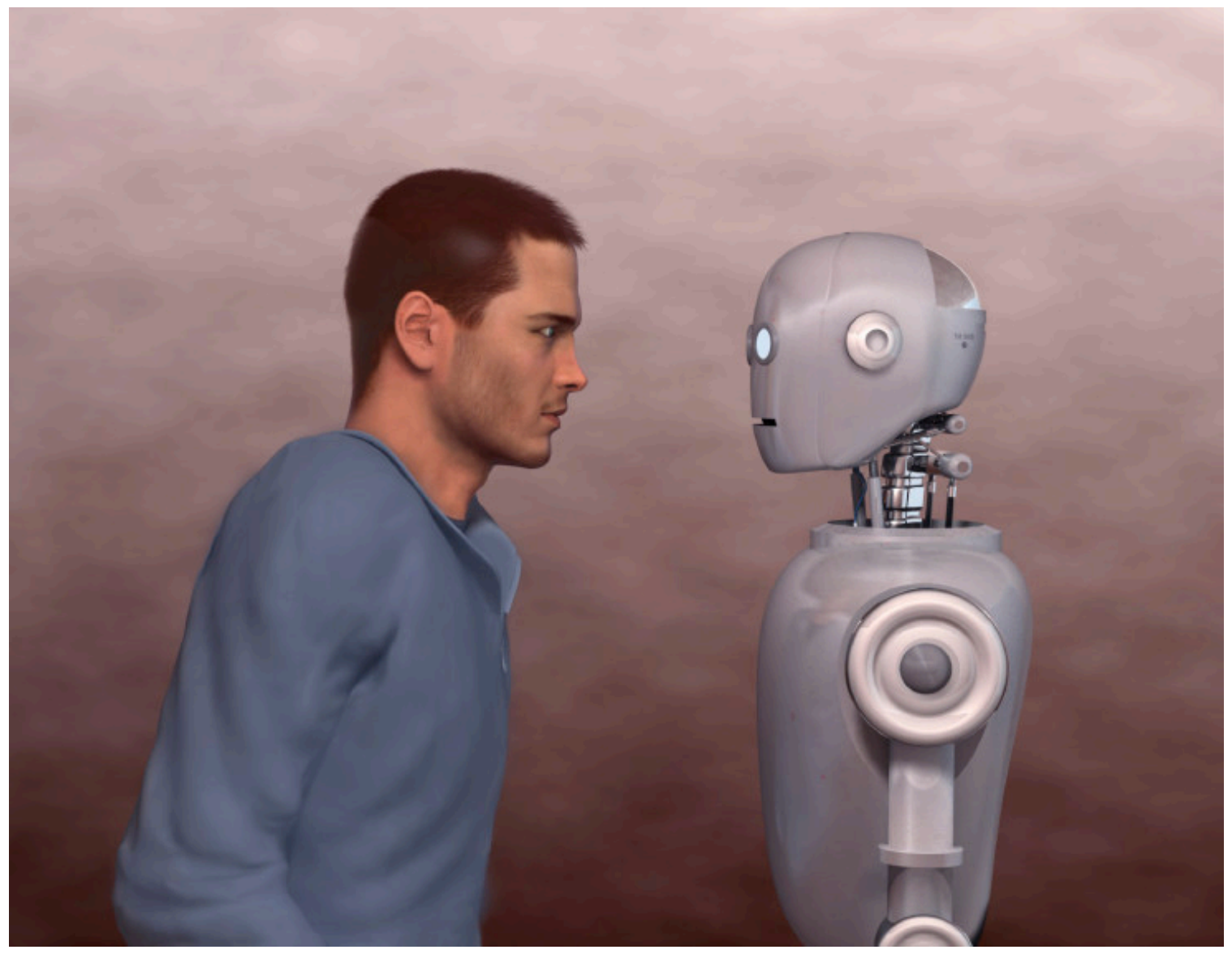

Figure 12: Humaoid (or droid) robot (Brockwell, 2015)

Although humanoids (or droids) would have the ability to act, think and reason

like a healthcare worker or a nurse, it is also important to raise the question if these robots would have the ability to make an ethical decision within a hospital setting (Mesko, 2015). This is a question that can only be answered if we do in fact: see humanoid (or droid) robots within hospitals in the future. 
Humanoids (or droids) as mentioned above will not only be an huge advancement for robotics, but also for Wayfinding. Advancements in hospital robotics are in fact, assisted by revolutionary advances in Wayfinding systems, which allow these robots to function and navigate throughout hospitals and of course perform their duties. Without Wayfinding systems implemented in these robots, these robots have no purpose. Robots like TUG and Homer, are already using sophisticated technologies like RFID, RFID tags, programmed maps, sensors and Wi-Fi to navigate their way throughout hospitals. Although humanoid (or droid) robots are still very much a concept that is being explored by designers and engineers, these robots will require even more advanced Wayfinding methodologies than TUG and Homer to function just as or even better than healthcare workers and nurses. However, to envision a Wayfinding system for robots so superior, designers and engineers would have to create a Wayfinding system that can mimic virtually any human movement. The thought of having a robot with not only a higher level of intelligence but also this level of mobility will only intensify the need for more robots in hospitals.

\subsection{Conclusions}


Advances in robotics and Wayfinding have indeed changed healthcare particularly in hospitals. Hospitals are very complex environments that provide healthcare services to patients 24 hours a day and 7 days a week. Everyday there are many tasks and procedures that need to completed and taken care of. Designers and engineers are constantly coming up with new solutions to assist hospitals with these tasks and procedures. Robots have been implemented throughout hospitals for quite some time to assist in many medical surgeries. However, now, hospitals are looking into adapting new innovative technologies like the TUG and Homer to complete various tasks throughout the hospital (O'Connor).

Although robots like TUG and Homer are sophisticated robotic technologies with superior Wayfinding systems, these robots are raising the concern of many healthcare workers and nurses jobs in hospitals (Ross, 2012). Also, many patients feel that implementing more robots like TUG and Homer will eventually take the humanity out of healthcare. Additionally, questions are being raised if robots like TUG and Homer are in fact, safe to operate within hospital settings.

There is defiantly an interesting divide between having sophisticated robotic technologies with superior Wayfinding systems like TUG and Homer, compared 
to healthcare workers and nurses who carry out tasks and procedures in a traditional manner. On one hand, hospitals want new and innovative robotic technologies that do not only lower hospitals costs, but complete tasks without any distractions and hitches (How It Works, 2016). On the other hand, healthcare workers and nurses feel that robots like TUG and Homer cannot provide the same assistance and care for patients as they can.

However, robotic technologies with superior Wayfinding systems like TUG and Homer are constantly being implemented in various hospitals and are continuing to explored to create even more advanced robots that will eventually be seen roaming throughout hospitals. Although robotic technologies like TUG and Homer are still evidentially new, it will only be a matter of time, until we figure out if robots like TUG and Homer are in fact safe to be operating in hospitals, and if these robots do end up replacing healthcare workers and nurses. More research is needed. 


\section{References}

Angelo, J. A. (2007). Robotics: A Reference Guide to the New Technology. Libraries Unlimited.

Bridges, H. (2015, June 19). Robotic rounds: Would you take your meds from a robot? Retrieved June 20, 2016, from http://nuviun.com/content/roboticrounds-would-you-take-your-meds-from-a-robot

Brockwell, H. (Ed.). (2015, October 28). Interview: Dr Kevin Curran on how robots will coexist with humans. Retrieved June 14, 2016, from http://www.gadgette.com/2015/10/28/Interview-dr-kevin-curran-on-how-robotswill-coexist-with-humans/

Caldwell, D. G. (1996) Advanced Robotics \& Intelligent Machines (Vol. 51) (J. O. Gray, Ed.) IET.

Corrales, A., Malfaz, M., \& Salichs, M. A. (n.d.). Signage system for the navigation of autonomous robots in indoor environments. 1-9. Retrieved February 12, 2016.

Davidow, W. H., \& Malone, M. S. (2014, December 10). What Happens to Society When Robots Replace Workers? Retrieved July 27, 2016, from http://hbr.org/2014/12/what-happen-to-society-when-robots-replace-workers

Davidson, S. (2015, October 18). Fully digital Humber River Hospital opens in Toronto. Retrieved May 25, 2016, from http://toronto.ctvnews.ca/fully-digitalhumber-river-hospital-open-in-toronto-1.2615880

Del Prado, G. M. (2015, September 30). This is the best career option if you don't want a robot to take your job. Retrieved July 28, 2016, from http://www.techinsider.io/nursing-jobs-wont-be-taken-by-robots-2015-9

El-Rabbany, A. (2002). Introduction to GPS: The Global Positioning System (Vol. 0, p.176). Artech House. 
Farman, J. (2013). The Mobile Story: Narrative Practices with Locative Technologies. Routledge.

Gallo, A. (n.d.). Wi-Fi fills gap in indoor location tracking market. Retrieved July 24, 2016, from http://www.searchnetworking.techtarget.com/tip/Wi-Fi-fills-gapin-indoor-location-tracking-market

Gibson, D. (2009). The Way-finding Handbook: Information Design for Public Places (p. 152). Princeton Architectural Press.

Grant, A. E., \& Meadows, J. H. (Eds.). (2012). Communication Technology Update and Fundamentals (13 ${ }^{\text {th }}$ ed.). Taylor \& Friends.

Hanson, D. (2011, April 1). Why We Should Build Humanlike Robotics. Retrieved July 27, 2016, from

http://spectrum.ieee.org/automaton/robotics/humanoids/why-we-should-buildhumanlike-robots

Hassler, S. (2016, June 1). Would You Trust a Robot to Give Your Grandmother Her Meds? Retrieved July 31, 2016, from

http://spectrum.ieee.org/robotics/artifical-intellegience/would-you-trust-a-robotto-give-your-grandmother-her-meds

Havenstein, H. (2007, February 27). RFID-Enabled Robots Track Medical Equipment. Retrieved June 8, 2016, from http://www.pcworld.com/article/129391/article.html

Hayzlett, G. (2015). Beyond the Sign. American Planning Association, 3335. Retrieved January 24, 2016, from http://www.rmimpr.com/wpcontent/uploads/2015/08/Final-APA-Article-April-2015.pdf

High-Tech 'TUG' Robots Will Do Heavy Lifting at Mission Bay. (n.d). Retrieved June 8 2016, from http://www.ucsfmissonbayhospitals.org/articles/high-techtug-robots-do-heavy-lifting-at-mission-bay.html

How It Works. (2016). Retrieved June 30, 2016, from http://www.aethon.com/tug/how-it-works

Jacobson, R., \& Jacobson. (2000). Information Design (R.E. Jacobson. Ed.). MIT Press. 
Kutscher, B. (2016, April 30). Inside North America's first all-digital hospital. Retrieved July 28, 2016, from http://www.modernhealthcare.com/article/20160430/MAGAZINE/304309981

Jones, W. (2010) Keeping Found Things Found: The Study and Practice of Personal Information Management. Morgan Kaufmann.

Lee, J. (2013, May 25). Robots get to work: More hospitals are using automated machines, but jury's still out on success. Retrieved June 14, 2016, from http://www.modernhealthcare.com/article/20130525/MAGAZINE/305259957

Melanson, A. (2016, July 20). Intralogistics is the Secret to a Smart Supply Chain. Retrieved June 25, 2016, from http://www.aethon.com/intralogistics-is-thesecret-to-a-smart-supply-chain

Mesko, B. (2015). My Health: Upgraded: Revolutionary Technologies To Bring A Healthier Future. Webicina Kft.

Niku, S. (2010). Introduction to Robotics. John Wiley \& Sons.

O'Connor, M. C (n.d). Aethon Adds RFID to Robotic Hospital Helpers. Retrieved may 25, 2016, from http://www.rfidjournal.com/articles/view?3330

Poulin, R. (2012). Graphic Design and Architecture, A 20 th Century History: A Guide to Type, Image, Symbol, and Visual Storytelling in the Modern World. (p. 272). New York: Rockport.

Powell, L. (2015, July 6). Silence, please. This is an airport. CNN. Retrieved January 21, 2016, from http://www.cnn.com/2015/07/06/travel/silentairports/index.html

Puffer, B.T. (2011). Assistive Navigation: A Study Incorporating the Use of WiFi Networks to Provide Realtime, Interactive Wayfinding in Enclosed Structures for Individuals Who Have Visual Impairments. 1-144. Retrieved February 12, 2016.

Reffat, R. M. (n.d.). Hospitals of the Future using Advanced Technologies. 1-11. Retrieved February 12, 2016. 
Ross, G. (2012, April 10). Robotics in healthcare: Challenges and opportunities. Retrieved June 14, 2016, from http://medicaldesign.com/archive/roboticshealthare-challenges-and-opportunities-0

Samani, N., Haji Babai., L., Delavar, M., Malek, M., \& Frank, A. (n.d.). An Agentbased Indoor Way-finding Based on Digital Sign System. 1-11. Retrieved February 12, 2016.

Simon, M. (2015, October 2). This Incredible Hospital Robot Is Saving Lives. Also, I Hate It. Retrieved June 14, 2016, from http://www.wired.com/2015/02/incredible-hospital-robot-saving-lives-also-hate/

Snow, J. (2016, February 11). Medicine under fire: How to hack a hospital. Retrieved June 16, 2016, from http://blog.kaspersky.com/hackedhospital/11296/

Su, N. M., Liu, L. S., \& Lazar, A. (n.d). Mundanely Miraculous: The Robot in Healthcare. 1-10. Retrieved July 28, 2016, from http://homes.soic.indiana.edu//sliu/papers/p391-su.pdf

The First Robot Operated Hospital In North America Has Finally Opened. (2015, October 21). Retrieved June 8, 2016, from http://futurism.com/the-first-robotoperated-hospital-in-north-america-has-just-opened-its-doors/

What is Wifi and How Does it Work. (2016, August). Retrieved May 30, 2016, from http://ccm.net/faq/298-what-is-wifi-and-how-does-it-work

Zetter, K. (2014, April 25). It's Insanely Easy to Hack Hospital Equipment. Retrieved June 16, 2016, from http://www.wired.com/2014/04/hospitalequipment-vulneable/ 